\title{
A progressive cone-rod dystrophy and amelogenesis imperfecta: a new syndrome
}

\author{
I K JALILI* AND N J D SMITH $\dagger$
}

From *St John Ophthalmic Hospital, Jerusalem; and †King's College School of Medicine and Dentistry,. Denmark Hill, London SE5 8RX.

SUMmARY Twenty-nine members of an extended Arab family from the Gaza Strip were foundo to be affected with cone-rod dystrophy and amelogenesis imperfecta, inherited in an autosomaler recessive manner.

\section{Medical history}

All affected members suffered from photophobia and nystagmus, starting in the first two years of life, and achromatopsia. There was no night blindness. All dentate members had abnormal, discoloured teeth.

\section{Clinical examination}

OCULAR

Visual acuities ranged from $6 / 36$ to no perception of light, and a marked pendular nystagmus was present in all cases. The earliest fundus abnormality, observed in both eyes of a three month old child, was a round area of retinal pigment epithelial atrophy at the macula giving rise to an early bull's eye appearance. As the condition progressed, there was more extensive atrophy of the retinal pigment epithelium at the macula (fig 1) and the equator. In the most advanced cases (aged 45 and 50 years), there was extensive chorioretinal atrophy of the macula and pigment deposition in the equatorial and peripheral retina which also involved the macula (bone corpuscle appearance). The optic nerve had appeared normal in the early stages, but during the course of the disease there was progressive optic atrophy leading to a pale, waxy optic disc.

\section{DENT A L}

Tooth morphology was abnormal in all the members with retinal dystrophy (fig 2 ). The surfaces of the teeth were rough and became stained soon after eruption and rapidly developed a dark brown discolouration. The enamel layer was absent in 23 subjects and grossly hypoplastic in four. The

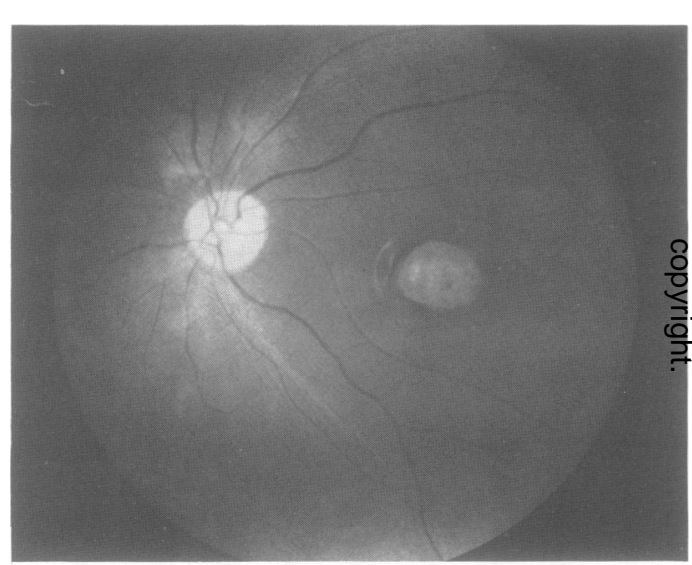

FIG 1 Fundus of a 10 year old male patient showing macular chorioretinal atrophy giving the appearance of 'macular coloboma'.

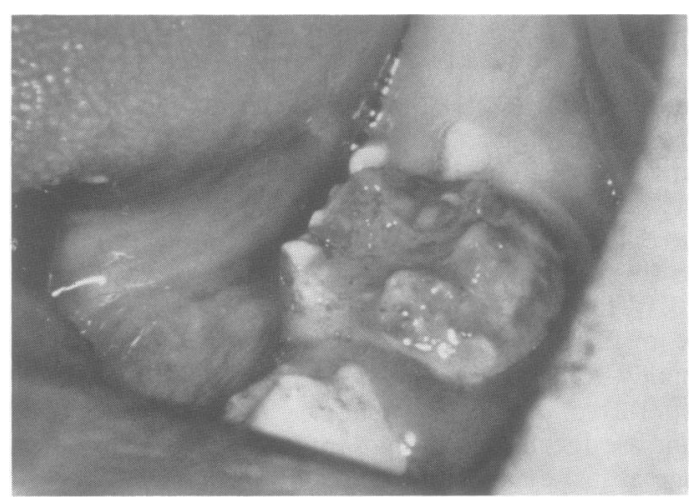

FIG 2 A lower left first permanent molar showing the absence of enamel, the unusual morphology, and th staining. 


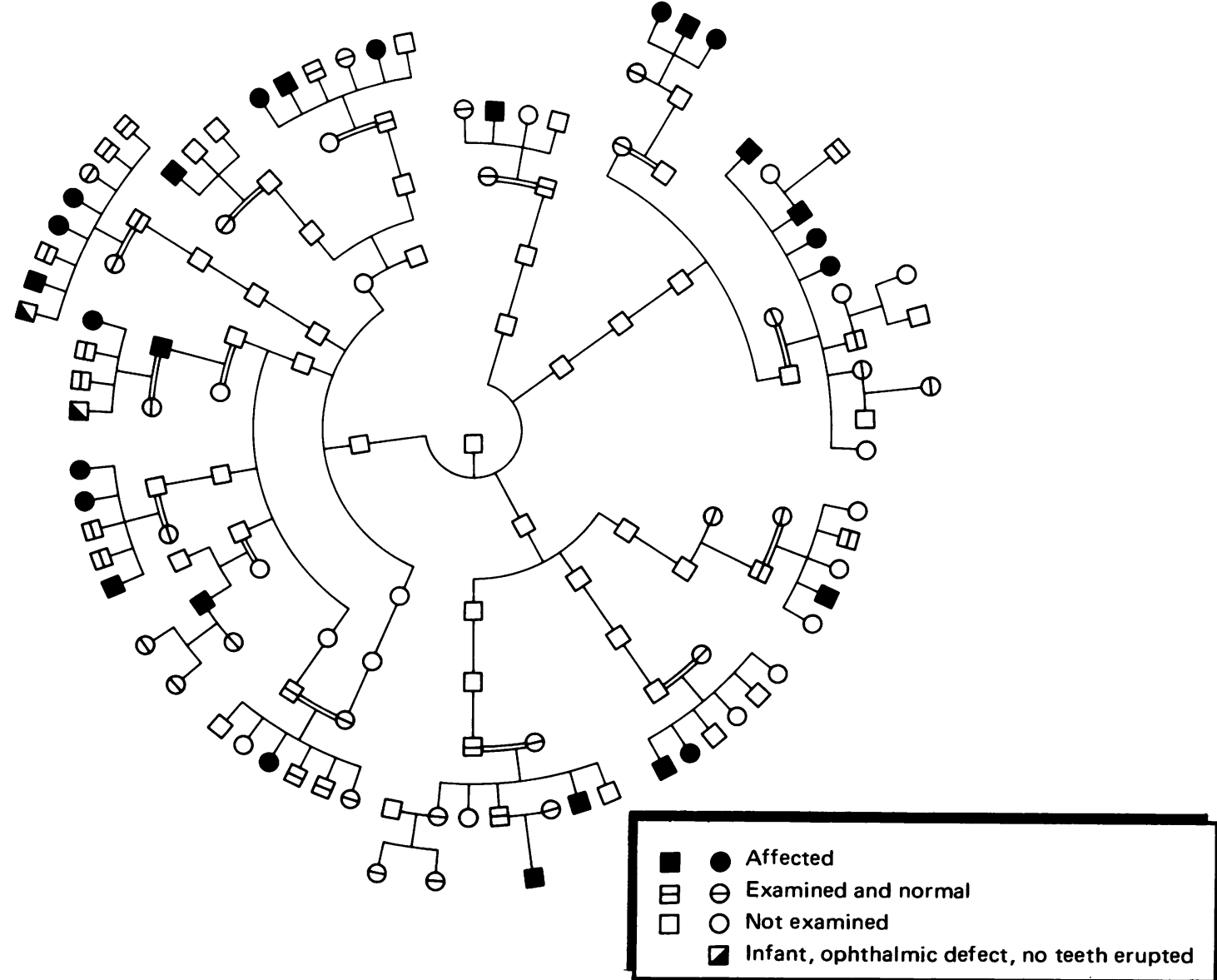

FIG 3 This is a simplified version of a much fuller pedigree which shows the exact relation of the husbands and wives. There was consanguinity in all but one branches of the family.

remaining two cases were babies who had no teeth erupted at the time of examination.

\section{OTHER}

There was neither auditory impairment nor any other systemic abnormality.

\section{Investigations}

Colour vision was tested with Ishihara pseudoisochromatic plates and total achromatopsia was found.

Peripheral fields (Goldmann perimetry) were full in the early cases but progressive restriction occurred with advancement of the rod involvement.

Electrodiagnostic tests were performed on three male patients aged 12,17 , and 19 with varying severity of disease with the following results.

EOG (normal $>180 \%$ ) was normal early in the disease (12 years, $250 \%$ right, $275 \%$ left), becoming subnormal (17 years, $150 \%$ right, $146 \%$ left) and flat in the more advanced cases (19 years, $117 \%$ right, $120 \%$ left).

Flash scotopic ERG of the first patient showed normal a wave and mildly reduced $b$ wave and the last two patients had severely reduced $a$ and $b$ waves. Flicker response (at $30 \mathrm{~Hz}$ ) was absent in all three patients.

Dental panoramic tomography was performed. Either no enamel or only vestiges of the enamel cap were present in the unerupted as well as the erupted teeth.

Three teeth from different patients were extracted 
for sound clinical reasons. Macroscopical examination of the extracted teeth showed small areas of enamel at the cervical margin of the teeth.

Microscopically, ground sections showed an absence of enamel except cervically, where there was enamel of normal thickness irregularly broken away towards the occlusal surface. The features were reported as being compatible with a diagnosis of amelogenesis imperfecta of the hypomineralised (hypocalcified) type.

\section{Genetics}

The family pedigree is shown in fig 3 . Fourteen sub-kindreds were examined between January 1986 and March 1987. There were 13 consanguineous marriages, ranging from first to third cousins. Analysis of the pedigree, compiled for seven generations of the kindred, indicated an autosomal recessive mode of inheritance. Among the living sibs examined (84, M:F ratio 1.1:1), the percentage of the affected examined (29) of the total number of sibs was $34.5 \%$ (M:F ratio $1.23: 1$ ). A further three branches of the family living abroad were said to have five out of 11 sibs affected with this oculodental disorder. Variable expression is found in some subkindreds.

\section{Discussion}

Cone-rod dystrophies are a relatively rare group of progressive cone disorders. Initially they present, during the first two decades of life, with symptoms of cone involvement with progressive loss of central visual acuity and colour vision, photophobia, and nystagmus, and absence of night blindness. Rod involvement takes place later. Night blindness is relatively rare but when present it is usually associated with the more advanced stage of the dystrophy. Clinically there is a characteristic bull's eye macular lesion, markedly decreased or absent photopic ERG responses, mildly decreased scotopic ERG responses, and, in the later stages, peripheral retinal pigmentation with retinal arteriolar attenuation. They may be inherited as an autosomal dominant or autosomal recessive trait. The latter is more severe and has a worse visual prognosis. ${ }^{1}$

Cone-rod dystrophy should be differentiated from rod monochromatism, pericentral and sine forms of retinitis pigmentosa, Stargardt's disease, central areolar choroidal dystrophy, chloroquine retinopathy, and congenital optic atrophy.

Amelogenesis imperfecta ${ }^{2}$ is a generic term for a number of different hereditary abnormalities of dental enamel formation. Three different types are described: hypoplastic, hypocalcified (hypomineral-

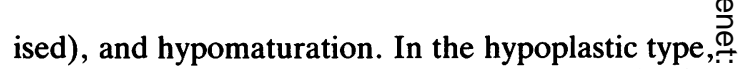
the enamel is not of normal thickness, while in the $\Rightarrow$ hypocalcified type the enamel is of normal thickness $\stackrel{\vec{F}}{+}$ at eruption, but is softer than normal and wears away rapidly once the teeth erupt into the mouth. In the hypomaturation type, the defect appears to be $\frac{\rho}{\widetilde{\sigma}}$ associated with the enamel root sheaths. The enamel is of normal thickness at the time of eruption, but is softer than normal and tends to chip away leaving an irregular surface.

There was an unequivocable association between $\vec{\omega}$ the retinal dystrophy and the amelogenesis imper-o fecta. Members of the family who had normal vision also had teeth which showed a normal pattern of enamel formation, except that in many cases the typical staining of endemic fluorosis was present. $\overrightarrow{-}$

The association of enamel defects and ocular $\omega$ disease has been documented in oculodentodigitalo dysplasia (oculodento-osseous dysplasia) and은 Rieger's syndrome ${ }^{3}$ but these are different from the condition reported here. Bateman et $\mathrm{l}^{4}$ described a $Z$ series of unrelated patients, two of whom (cases $7 \AA$ and 13) had a combination of ocular defects, hearing loss, and enamel hypoplasia. The retinal dystrophy, as described, does not appear identical to that in our $\overrightarrow{.}$ series, and none of our patients had any evidence $8 f_{0}^{\circ}$ hearing loss.

In our pedigree, the 29 who were examined haळi. widespread dystrophy affecting the retina and in $\bar{z} 7 \leq$ of these the enamel was affected. The other two were infants with no erupted teeth. Retinal coneš were primarily affected with later rod involvement疋 (cone-rod dystrophy). This association has not been $\overrightarrow{\vec{B}}$ previously reported. 35

We would like to thank Professor Barrie Jayo for help and advice on the scientific aspects of this paper and Dr Lee Chumbley and the Order of Sta John of Jerusalem for providing the facilities ando resources for this work.

\section{References}

1 Krill AE, Deutman AF, Fishman M. The cone degeneration음 Doc Ophthalmol 1973;35:1-80.

2 Witkop CJ, Sauk JJ. Heritable defects of enamel. In: Stewart E Prescott GH, eds. Orofacial genetics. St Louis: Mosby, 1976.

3 McKusick VA. Mendelian inheritance in man. 7th ed. Balti- $N$ more: Johns Hopkins University Press, 1986.

4 Bateman JB, Reidner ED, Levin LS, Maumenee IH. Am N Ophthalmol 1980;90:755-67.

5 Winter RM, Baraister M, Douglas JM. A computerised data $\omega$ base for the diagnosis of rare dysmorphic syndromes. $J$ Me Genet 1984;21:121-3.

Correspondence and requests for reprints to Professor N J D Smith, Department of Dentaio Radiology, Dental School, Denmark Hill, Londono SE5 8RX. 